\title{
3 Research Square

\section{Clinical characteristics, imaging phenotypes and events free survival in hypertensive Takayasu arteritis population}

\section{Ying Sun}

Zhongshan Hospital Fudan University

\section{Sifan Wu}

Zhongshan Hospital Fudan University

\section{Yujiao Wang}

Zhongshan Hospital Fudan University

\section{Rongyi Chen}

Zhongshan Hospital Fudan University

\section{Qingrong Huang}

Zhongshan Hospital Fudan University

\section{Lili Ma}

Zhongshan Hospital Fudan University

\section{Huiyong Chen}

Zhongshan Hospital Fudan University

Lindi Jiang ( $\nabla$ zsh-rheum@hotmail.com )

Zhongshan Hospital Fudan University https://orcid.org/0000-0001-7747-7788

\section{Research article}

Keywords: Takayasu arteritis, hypertension, hypertensive severity, imaging phenotype, blood pressure control status, events free survival

Posted Date: May 17th, 2021

DOl: https://doi.org/10.21203/rs.3.rs-525884/v1

License: (c) (1) This work is licensed under a Creative Commons Attribution 4.0 International License. Read Full License

Version of Record: A version of this preprint was published at Arthritis Research \& Therapy on July 21 st, 2021. See the published version at https://doi.org/10.1186/s13075-021-02579-8. 


\section{Abstract \\ Background}

Hypertension occurred in $30-80 \%$ of Takayasu arteritis (TAK) patients around the world and the occurrence of hypertension might worsen the disease prognosis. This study aimed to investigate the clinical characteristics and imaging phenotypes, as well as their associations with events free survival (EFS) in hypertensive TAK population.

\section{Methods}

This current research was based on a prospectively on-going observational cohort-the East China Takayasu Arteritis (ECTA) cohort, centered in Zhongshan Hospital, Fudan University. Totally, 204 hypertensive TAK patients were enrolled between January 2013 and December 2019. Clinical characteristics and imaging phenotypes of each case were evaluated and their associations with the EFS by the end of August 30, 2020 were analyzed.

\section{Results}

Severe hypertension accounted for $46.1 \%$ of the entire population. Three specific imaging phenotypes were identified: Cluster 1: involvement of the abdominal aorta and/or renal artery (27.5\%); Cluster 2: involvement of the ascending aorta, thoracic aorta, and the aortic arch and/or its branches (18.6\%); and Cluster 3: combined involvement of Cluster 1 and 2 (53.9\%). Clinical characteristics, especially hypertensive severity, differed greatly among three imaging clusters. In all, 187 patients were followed-up for a median of 46 (9-102) months; 127 (67.9\%) cases did not experience any events, while 72 events were observed in 60 patients. The overall blood pressure control rate was $50.8 \%$, and the EFS was $67.9 \%$ by the end of the follow-up. Multivariate Cox regression indicated that controlled blood pressure $(\mathrm{HR}=$ $2.13,95 \% \mathrm{Cl} 1.32-3.74)$, Cluster 1 ( $\mathrm{HR}=0.69,95 \% \mathrm{Cl} 0.48-0.92)$ and Cluster $3(\mathrm{HR}=0.72,95 \% \mathrm{Cl} 0.43-$ 0.94) imaging phenotype was associated with the EFS. Kaplan-Meier curves showed that patients with controlled blood pressure showed better EFS $(p=0.043)$. Furthermore, patients had controlled blood pressure and Cluster 1 phenotype was set as reference, better EFS was observed in patients with controlled blood pressure and Cluster 2 phenotype $(\mathrm{HR}=2.21,95 \% \mathrm{Cl} 1.47-4.32)$, while those had uncontrolled blood pressure and Cluster 1 phenotype $(\mathrm{HR}=0.64,95 \% \mathrm{Cl}: 0.52-0.89)$ and those had uncontrolled blood pressure and Cluster 3 phenotype ( $\mathrm{HR}=0.83,95 \% \mathrm{Cl}: 0.76-0.92)$ suffered worse EFS.

\section{Conclusion}

Blood pressure control status and imaging phenotypes showed significant effects on the EFS for hypertensive TAK. 


\section{Background}

Takayasu arteritis (TAK) is a chronic inflammatory large-vessel vasculitis that primarily affects the aorta and its main branches $[1,2,3]$. Hypertension is a particularly important complication in patients with TAK $[4,5,6]$. According to previous reports, hypertension occurred in $33-83 \%$ of patients with TAK from different areas of the world, with younger age of disease onset [7-10]. The occurrence of hypertension could severely worsen TAK prognosis and may be a significant prognostic predictor of outcomes [11]. Furthermore, uncontrolled blood pressure in hypertensive TAK patients was associated with a higher five years all-cause mortality risk than that in the healthy population, despite effective control of disease activity $[12,13]$. Thus, comprehensive understanding of the disease characteristics of hypertensive TAK is very essential.

To our knowledge, few studies have focused on hypertension in the TAK population up to date. In a previous research, we reported that in patients with TAK-related renal artery stenosis, the prevalence of hypertension was up to $60 \%$, with $30 \%$ refractory hypertensive cases [14]. Except renal artery stenosis, the involvement of abdominal aorta, as well as severe aortic regurgitation (AR) also could cause hypertension in $\operatorname{TAK}[15,16,17]$. Nevertheless, data describing whether there are specific imaging features in hypertensive TAK patients as well as the associations with disease prognosis were still lacking.

Thus, this study was designed to investigate the clinical characteristics and specific imaging phenotypes of TAK patients with hypertension and to point out the associations of the clinical characteristics and imaging phenotypes with the events free survival (EFS).

\section{Methods}

\section{Study design and subjects}

The present study was based on a prospectively on-going observational cohort-the East China Takayasu Arteritis (ECTA) cohort, centered in Zhongshan Hospital, Fudan University, Shanghai, China. All patients enrolled into the ECTA cohort had a confirmed diagnosis of TAK based on the 1990 American College of Rheumatology (ACR) criteria [18]. The demographic, clinical, laboratory, and treatment data were collected at baseline and each visit. The follow-up frequency was once a month in the active phase and once every 3 months in the remission phase. Disease activity was assessed using the National Institutes of Health $(\mathrm{NIH})$ criteria [19]. The clinical data of all enrolled patients were recorded and stored in a unified electronic database (REDCap database system, https://redcap.zs-hospital.sh.cn).

In all, 204 TAK patients with hypertension, were enrolled in to the current research from the ECTA cohort between January 2013 and December 2019. Clinical characteristics and imaging features of each case were evaluated by professional rheumatologists. The main outcome of the investigation was the EFS by the end of August 30, 2020 (Fig. 1). Associations of the clinical and imaging features with the EFS were analyzed. The study was performed in accordance with the tenets of the Helsinki Declaration and its 
amendments. The study protocol was approved by the Ethics Review Board of Zhongshan Hospital (B2013-115(3)). Written informed consent was obtained from all patients.

\section{Blood pressure measurement}

Upper-limb blood pressure was measured in all patients. Blood pressure measurement of the four limbs, and ankle brachial index (ABI) was performed in 92/204 (45.1\%) patients by using a noninvasive blood pressure monitor (BP-203RPEIII, Omron Healthcare Co., Ltd., Tokyo, Japan). For those without subclavian artery involvement, the reading from the arm with the higher value was used as the reference measurement. For patients with unilateral subclavian artery involvement, the reading from the unaffected side was analyzed, while for those with bilateral subclavian artery involvement, higher values of blood pressure of the lower limbs were used for analysis.

\section{Classification of hypertensive severity and blood pressure control status}

The severity of hypertension was classified as previously reported [20]: (i) mild: a brachial pressure of 140-159 mmHg systolic or 90-99 mmHg diastolic; (ii) moderate: a brachial pressure of $160-179 \mathrm{mmHg}$ systolic or $100-109 \mathrm{mmHg}$ diastolic; and (iii) severe: a brachial pressure of $\geq 180 \mathrm{mmHg}$ systolic or $\geq$ $110 \mathrm{mmHg}$ diastolic.

Blood pressure control status was classified as: (i) controlled: systolic blood pressure (SBP) $<140 \mathrm{mmHg}$ and diastolic blood pressure (DBP) $<90 \mathrm{mmHg}$; (ii) improved: SBP $\geq 140 \mathrm{mmHg}$, but decreased by $\geq 20$ $\mathrm{mmHg}$ and/or DBP $\geq 90 \mathrm{mmHg}$, but decreased by $\geq 10 \mathrm{mmHg}$; and (iii) failure: failed meeting the abovedefined criteria. Refractory hypertension was defined as a brachial pressure $\geq 160 \mathrm{mmHg}$ systolic or $\geq 90$ $\mathrm{mmHg}$ diastolic pressure despite maximal doses of three antihypertensive drugs for at least one month $[14,21]$.

\section{Imaging measurements}

Imaging assessments, mainly the whole-body enhanced magnetic resonance angiography (MRA) or computed tomography angiography (CTA) were performed at the time of enrollment. Angiography findings were classified according to the classification by Numano et al. in 1996 [22].

Diagnosis of AR was confirmed by echocardiography according to the guideline of the American Society of Echocardiography [23]. The severity of AR was evaluated by echocardiography as previously described $[24,25]$.

\section{Outcomes}

Patients, who completed at least 6 months follow-up, were included in the outcome analysis. The occurrence of any events during the follow-up included: (i) renal insufficiency including new occurrence, persistent insufficiency ( $\geq 6$ months) or deterioration of renal function ( $\geq 20 \%$ increase in creatinine concentration or $\geq 20 \%$ decrease in glomerular filtration rate (GFR); (ii) persistent refractory hypertension 
( $\geq 6$ months) or malignant hypertension; (iii) congestive heart failure including new occurrence or deterioration of heart function; (iv) new occurrence of cerebrovascular events; (v) arterial dissection or rupture of aneurysms; or (vi) TAK-related death (e.g., death caused by severe arterial stenosis or aortic dissection).

\section{Statistical analysis}

Categorical variables were summarized as counts and percentages and were compared using chi-square or Kruskal-Wallis tests. Continuous variables are presented as means \pm standard deviations (SD) or as medians with interquartile range (IQR), depending on the normality of distribution, and were compared using Student's t-tests, Wilcoxon tests, or one-way ANOVA. For variables with significant differences among three or more groups, pair-wise comparisons were further performed using Student's $t$-tests, Wilcoxon tests, or chi-square tests.

To identify specific imaging phenotypes for hypertensive TAK patients, 14 arteries including bilateral carotid arteries, brachiocephalic trunk, bilateral subclavian arteries, aortic arch, ascending aorta, thoracic aorta, pulmonary artery, abdominal aorta, bilateral renal artery, superior mesenteric artery and celiac axis were included in the cluster analysis by a two-step progress as described previously [26]. Individual arteries were clustered on the presence or absence of arteriographic lesions, and agglomerative, hierarchical clustering was performed. The cluster algorithm started with each individual artery as a single cluster. In successive iterations, the two nearest clusters were merged together on the basis of a measure of similarity to form a new, unique cluster. The process was repeated until all of the data were contained in one cluster. Tree dendograms were created to visualize cluster patterns.

Cox proportional hazards regression model was used to evaluate associations of imaging phenotypes and clinical characteristics with EFS during the follow-up by adjusting for age, sex, disease duration, disease activity, and received medications. Hazard ratios (HR) and $95 \%$ confidence intervals (Cls) were reported. The Kaplan-Meier method was used to plot the proportion distribution of EFS in the above subgroups over time with log-rank test. Statistical analyses were performed using SPSS 22.0 (IBM Corp., Armonk, New York, USA). Two-sided $p<0.05$ was considered to indicate statistical significance.

\section{Results}

\section{Patients general characteristics}

In total, $204(33 \%)$ patients (155 [76\%] female) suffered from hypertension in our cohort, with dizziness/headache $(88 / 204,43.1 \%)$, chest distress $(57 / 204,27.9 \%)$ and weakness $(58 / 204,28.4 \%)$ as the most common manifestations. According to the 1996 classification, Type V (112/204, 54.9\%) was the most common imaging type, followed by type IV (44/204, 21.6\%). The demographic and clinical characteristics were presented in Supplementary Table S1. 
In comparison with non-hypertensive patients, higher prevalence of renal insufficiency $(8.8 \%$ vs. $2.2 \%, p=$ $0.001)$ and heart failure $(11.8 \%$ vs. $5.8 \%, p=0.009)$ was observed in hypertensive patients. Imaging types and arterial involvement significantly differed $(p<0.001)$, showing higher prevalence of renal artery involvement $(56.9 \%$ vs. $13.3 \%, p<0.001)$ and abdominal aorta involvement $(51.5 \%$ vs. $22.7 \%, p<0.001)$ in patients with hypertension (Supplementary Table S1).

\section{Characteristics of patients with different hypertensive severity}

Mild, moderate, and severe hypertension was observed in 48 (23.5\%), 62 (30.4\%), and 94 (46.1\%) cases, respectively. Clinical characteristics of the three categories were summarized in Table 1. Age, sex, and disease duration were similar among the three categories. The prevalence of renal insufficiency $(p=$ $0.048)$, renal artery involvement $(p=0.043)$, as well as blood pressure control status $(p<0.001)$ significantly differed among the three hypertension categories. Patients with severe hypertension were more likely to experience failed control of blood pressure than those with mild $(12.6 \%$ vs. $4.6 \%, p<0.001)$ and moderate hypertension ( $12.6 \%$ vs. $5.3 \%, p=0.008)$, respectively. 
Table 1

Characteristics of patients with different hypertensive severity

$\begin{array}{llll}\text { Mild } & \text { Moderate } & \text { Severe } & \text { P- } \\ (\mathrm{N}=48) & (\mathrm{N}=62) & (\mathrm{N}=94) & \text { value }\end{array}$

\section{Demography}

Female $(\mathrm{n}, \%)$

Age (years, IQR)

Disease duration (months, IQR)

\section{Clinical manifestation ( $n, \%)$}

Systemic symptoms (fever, weakness, etc.)

Neurological symptoms (headache, amaurosis, etc.)

Cardiovascular symptoms (chest distress/pain, etc.)

\section{Baseline complications ( $\mathrm{n}, \%)$}

Renal insufficiency

Heart failure

Cerebral infarction

Lab test
$35(72.9 \%) \quad 47(75.8 \%) \quad 71(75.5 \%) \quad 0.752$

$40(27-49) \quad 35(27-46) \quad 37(24-48) \quad 0.271$

$24(2-96) \quad 34(6-96) \quad 24(3-102) \quad 0.513$
$19(39.6 \%) \quad 24(38.7 \%) \quad 34(36.2 \%) \quad 0.924$

$14(29.2 \%) \quad 26(41.9 \%) \quad 55(58.5 \%) \quad 0.544$

$9(18.8 \%) \quad 16(25.8 \%) \quad 32(34.1 \%) \quad 0.273$
BUN (mmol/L, IQR)

$\operatorname{SCr}(\mu \mathrm{mol} / \mathrm{L}, \mathrm{IQR})$

pro-BNP (pg/mL, IQR)

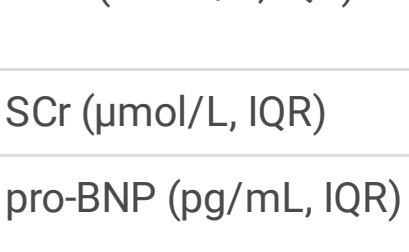

4.8 (3.7-

5.6)

$62(52-72)$

$100(53-$

423)

\section{Artery involvement $(n, \%)$}

Abdominal aorta
Renal artery

Thoracic aorta

Thoracic aorta
$2(4.2 \%))^{\#, *} \quad 5(8.1 \%)$

$4(8.3 \%)$

$2(4.2 \%)$
$7(11.3 \%)$

$2(3.2 \%)$
$11(11.7 \%)$

$13(13.8 \%)$

$5(5.3 \%)$
0.048

0.701

0.821

\begin{tabular}{lllll}
$20(41.7 \%)$ & $32(51.6 \%)$ & $53(56.4 \%)$ & 0.293 \\
\hline $20(41.7 \%)^{*}$ & $28(45.2 \%)^{\&}$ & $68(72.3 \%)$ & 0.043 \\
$10(20.8 \%)$ & $22(35.5 \%)$ & $32(34.1 \%)$ & 0.293
\end{tabular}

BUN: blood urea nitrogen; SCr: serum creatinine; BNP: brain natriuretic peptide; AR: aortic regurgitation; P-value: comparison among patients with different hypertensive severity; \#: $p<0.05$ for comparisons between patients with mild and moderate hypertension; *: $p<0.05$ for comparisons between patients with mild and severe hypertension; \&: $p<0.05$ for comparisons between patients with between patients with moderate and severe hypertension. 


\begin{tabular}{|c|c|c|c|c|}
\hline & $\begin{array}{l}\text { Mild } \\
(\mathrm{N}=48)\end{array}$ & $\begin{array}{l}\text { Moderate } \\
(\mathrm{N}=62)\end{array}$ & $\begin{array}{l}\text { Severe } \\
(N=94)\end{array}$ & $\begin{array}{l}\mathrm{P} \text { - } \\
\text { value }\end{array}$ \\
\hline Aortic arch & $5(10.4 \%)$ & $8(12.9 \%)$ & $11(11.7 \%)$ & 0.973 \\
\hline Carotid artery & $13(27.1 \%)$ & $26(41.9 \%)$ & $39(41.5 \%)$ & 0.115 \\
\hline Subclavian artery & $27(52.1 \%)$ & $33(53.2 \%)$ & $47(50.0 \%)$ & 0.744 \\
\hline \multicolumn{5}{|l|}{ Echocardiography (n, \%) } \\
\hline Severe AR & $3(6.3 \%)$ & $5(8.1 \%)$ & $11(11.7 \%)$ & 0.082 \\
\hline \multicolumn{5}{|l|}{ Blood pressure control status (n, \%) } \\
\hline Controlled & $26(60.5 \%)$ & $32(56.1 \%)$ & $37(42.5 \%)$ & 0.082 \\
\hline Improved & $15(34.9 \%)$ & $22(38.6 \%)$ & $39(44.8 \%)$ & 0.727 \\
\hline Failure & $2(4.6 \%) \star$ & $3(5.3 \%)^{\&}$ & $11(12.6 \%)$ & $<.001$ \\
\hline \multicolumn{5}{|l|}{ Events (n, \%) } \\
\hline $\begin{array}{l}\text { Persistent refractory or malignant } \\
\text { hypertension }\end{array}$ & $3(6.9 \%))^{\#, *}$ & $8(14.0 \%)$ & $16(18.4 \%)$ & 0.016 \\
\hline Renal insufficiency & $2(4.7 \%)$ & $4(7.0 \%)$ & $6(6.9 \%)$ & 0.322 \\
\hline Congestive heart failure & $2(4.7 \%)$ & $3(5.3 \%)$ & $2(2.3 \%)$ & 0.069 \\
\hline Cerebrovascular events & $1(2.3 \%)$ & $2(3.5 \%)$ & $2(2.3 \%)$ & 0.471 \\
\hline Events free survival by the end & $81.4 \% \star$ & $70.8 \%$ & $59.8 \%$ & 0.047 \\
\hline \multicolumn{5}{|c|}{$\begin{array}{l}\text { BUN: blood urea nitrogen; SCr: serum creatinine; BNP: brain natriuretic peptide; AR: aortic } \\
\text { regurgitation; P-value: comparison among patients with different hypertensive severity; \#: } p<0.05 \text { for } \\
\text { comparisons between patients with mild and moderate hypertension; } * \text { : } p<0.05 \text { for comparisons } \\
\text { between patients with mild and severe hypertension; \&: } p<0.05 \text { for comparisons between patients } \\
\text { with between patients with moderate and severe hypertension. }\end{array}$} \\
\hline
\end{tabular}

Immunosuppressive therapy including oral dose of glucocorticoids and the choice of immunosuppressors did not significantly differ the three hypertensive categories, while he medium kind of antihypertensive drugs and the medication choice differed. Patients with severe hypertension had higher revascularization rates than those with moderate hypertension ( $45.7 \%$ vs. $24.2 \%, p=0.008)$ (Supplementary Table S2).

\section{Characteristics of patients with different imaging phenotypes}

As significant differences of artery involvement were demonstrated in hypertensive patients, cluster analysis was further performed to explore new imaging phenotypes for hypertensive TAK population 
(Fig. 2): Cluster 1: involvement of abdominal aorta and/or renal artery ( $n=56,27.5 \%)$; Cluster 2:

involvement of ascending aorta, thoracic aorta, and/or the aortic arch and its branches ( $n=38,18.6 \%)$; and Cluster 3: combined involvement of Cluster 1 and Cluster $2(n=110,53.9 \%)$. The clinical characteristics of patients with different imaging phenotypes were shown in Table 2. 
Table 2

Characteristics of hypertensive patients with different imaging phenotypes

$\begin{array}{llll}\text { Cluster } 1 & \text { Cluster } 2 & \text { Cluster } 3 & \begin{array}{l}\text { P- } \\ \text { value }\end{array} \\ (\mathrm{N}=56) & (\mathrm{N}=38) & (\mathrm{N}=110) & \end{array}$

\section{Demography}

Female (n, \%)

Age (years, IQR)

Disease duration (months, IQR)

\section{Clinical manifestation $(n, \%)$}

Systemic symptoms (fever, weakness, etc.)

Neurological symptoms (headache, amaurosis, etc.)

Cardiovascular symptoms (chest distress/pain, etc.)

\section{Baseline complications ( $\mathrm{n}, \%)$}

Renal insufficiency

Heart failure

Cerebral infarction

Lab test

BUN (mmol/L, IQR)

$\operatorname{SCr}(\mu \mathrm{mol} / \mathrm{L}, \mathrm{IQR})$

pro-BNP (pg/mL, IQR)

\section{Hypertensive severity ( $n, \%)$}

Moderate

$18(32.1 \%)$

$5(8.9 \%)^{\#}$

$1(1.8 \%)^{\#, *}$

$1(1.8 \%)^{\star}$

$34(59.6 \%)^{*} \quad 30(78.9 \%) \quad 91(82.7 \%) \quad 0.007$

$27(20-39)^{\#} \quad 42(32-54)$

$39(27-49)<$

0.001

$12(3-56)$

$33(4-72)$

$30(4-120)$

0.334

$15(26.8 \%)$

$17(44.7 \%)$

45 (40.9\%)

0.320

$17(30.3 \%)$

21 (55.3\%)

$51(46.4 \%)$

0.461

$10(17.9 \%)^{\#}$

$16(42.1 \%)$

31 (28.2\%)

0.010

$1(2.6 \%)^{\&}$

$12(10.9 \%)$

0.046

$5(13.2 \%)$

$18(16.4 \%)$

0.013

0

$8(7.3 \%)$

0.007

Cluster 1: involvement of abdominal aorta and/or renal artery; Cluster 2: involvement of ascending aorta, thoracic aorta, aortic arch and its branches; Cluster 3: combined involvement of Cluster 1 and Cluster 2; BUN: blood urea nitrogen; SCr: serum creatinine; BNP: brain natriuretic peptide; AR: aortic regurgitation; P-value: comparison among patients with different imaging phenotypes; \#: $p<0.05$ for comparisons between patients with Cluster 1 and Cluster 2 phenotype; *: $p<0.05$ for comparisons between patients with Cluster 1 and Cluster 3 phenotype; and; \&: $p<0.05$ for comparisons between patients with Cluster 2 and Cluster 3 phenotype. 


\begin{tabular}{|c|c|c|c|c|}
\hline & $\begin{array}{l}\text { Cluster } 1 \\
(\mathrm{~N}=56)\end{array}$ & $\begin{array}{l}\text { Cluster } 2 \\
(\mathrm{~N}=38)\end{array}$ & $\begin{array}{l}\text { Cluster } 3 \\
(\mathrm{~N}=110)\end{array}$ & $\begin{array}{l}\mathrm{P}- \\
\text { value }\end{array}$ \\
\hline Severe & $26(46.4 \%)$ & $10(26.3 \%)^{\&}$ & 58 (52.7\%) & 0.014 \\
\hline \multicolumn{5}{|l|}{ Echocardiography (n, \%) } \\
\hline Severe AR & $4(7.1 \%)^{\#}$ & $5(11.4 \%)$ & $10(9.1 \%)$ & 0.047 \\
\hline \multicolumn{5}{|l|}{ Blood pressure control status (n, \%) } \\
\hline Controlled & $17(36.2 \%)^{\#}$ & $26(74.3 \%)$ & $52(47.3 \%)$ & $\begin{array}{l}< \\
0.001\end{array}$ \\
\hline Improved & $25(53.2 \%)$ & $7(20.0 \%)$ & $44(40.0 \%)$ & 0.117 \\
\hline Failure & $5(10.6 \%)^{\#}$ & $2(5.7 \%)$ & $9(8.2 \%)$ & 0.032 \\
\hline \multicolumn{5}{|l|}{ Events (n, \%) } \\
\hline $\begin{array}{l}\text { Persistent refractory or malignant } \\
\text { hypertension }\end{array}$ & $9(19.1 \%)^{\#}$ & $3(8.6 \%)^{\&}$ & 15 (13.6\%) & 0.024 \\
\hline Renal insufficiency & $4(8.5 \%)$ & 0 & $8(7.3 \%)$ & 0.178 \\
\hline Congestive heart failure & $1(2.1 \%)^{\#}$ & $2(5.7 \%)^{\&}$ & $3(2.7 \%)$ & 0.017 \\
\hline Cerebrovascular events & $1(2.1 \%)$ & $1(2.9 \%)$ & $3(2.7 \%)$ & 0.428 \\
\hline Events free survival by the end & $59.6 \% \#$ & $80.0 \%$ & $67.6 \%$ & 0.049 \\
\hline \multicolumn{5}{|c|}{$\begin{array}{l}\text { Cluster 1: involvement of abdominal aorta and/or renal artery; Cluster } 2 \text { : involvement of ascending } \\
\text { aorta, thoracic aorta, aortic arch and its branches; Cluster } 3 \text { : combined involvement of Cluster } 1 \text { and } \\
\text { Cluster 2; BUN: blood urea nitrogen; SCr: serum creatinine; BNP: brain natriuretic peptide; AR: aortic } \\
\text { regurgitation; P-value: comparison among patients with different imaging phenotypes; \#: } p<0.05 \text { for } \\
\text { comparisons between patients with Cluster } 1 \text { and Cluster } 2 \text { phenotype; *: } p<0.05 \text { for comparisons } \\
\text { between patients with Cluster } 1 \text { and Cluster } 3 \text { phenotype; and; \&: } p<0.05 \text { for comparisons between } \\
\text { patients with Cluster } 2 \text { and Cluster } 3 \text { phenotype. }\end{array}$} \\
\hline
\end{tabular}

Besides sex $(p=0.007)$ and age $(p<0.001)$, the prevalence of baseline features, including renal insufficiency $(p=0.046)$, heart failure $(p=0.013)$, cerebral infarction $(p=0.007)$, severe hypertension $(p=$ $0.014)$, and severe AR ( $p=0.047)$ differed significantly among the three imaging phenotype clusters. The blood pressure control status also differed among the three clusters of imaging phenotypes. The Cluster 1 group had lower prevalence of hypertension control $(36.2 \%$ vs. $74.3 \%, p<0.001)$ and higher prevalence of failed hypertension control ( $10.6 \%$ vs. $5.7 \%, p=0.019)$ than Cluster 2 group (Fig. 3$)$. In addition, immunosuppressive therapy and the medium kind of antihypertensive drugs, as well as the medication choice did not significantly differ among patients with different imaging phenotypes (Supplementary Table S3).

\section{Events free survival by the end of the follow-up}


Totally, 187/204 (91.7\%) patients were followed-up for a median of 46 (9-102) months, among whom, 127 cases did not experience any events, with a medium follow-up duration of 36 (8-100) months. Seventy-two events (1-3 per person) were observed in 60 (32.1\%) patients, with a median follow-up duration of 48 (6-92) months. The events included persistent refractory or malignant hypertension (27, $14.4 \%)$, persistent or deteriorated renal insufficiency $(12,6.4 \%)$, heart dysfunction $(7,3.7 \%)$, cerebrovascular events $(5,2.7 \%)$, aortic dissection $(2,1.1 \%)$, abdominal aortic dissection $(2,1.1 \%)$, rupture of abdominal aortic aneurysm $(1,0.5 \%)$ and death $(4,2.1 \%$ : two due to heart dysfunction, one due to rupture of aortic dissection, and one due to cerebrovascular event after bypass surgery of thoracic and abdominal aorta).

The EFS by the end of the follow-up was $67.9 \%$ in the entire population, while it was $81.4 \%, 70.8 \%$, and $59.8 \%$ in patients with mild, moderate, and severe hypertension, respectively; $59.6 \%, 80.0 \%$, and $67.6 \%$ in patients with Cluster 1, Cluster 2, and Cluster 3 imaging phenotype, respectively; and $75.8 \%, 61.8 \%$, and $50.0 \%$ in patients with controlled, improved, and control failure hypertension, respectively (Tables 1 and 2).

\section{Associations of the clinical characteristics and imaging phenotypes with events free survival}

Multivariate Cox regression analysis, with adjustments of age, sex, disease duration, disease activity, and received medications, indicated that co-existence of severe $A R(H R=0.87,95 \% \mathrm{Cl}: 0.64-0.95, p=0.043)$, controlled blood pressure ( $\mathrm{HR}=2.13,95 \% \mathrm{Cl}: 1.32-3.78, \mathrm{p}=0.047)$, Cluster $1(\mathrm{HR}=0.69,95 \% \mathrm{Cl}: 0.48-$ $0.92, p=0.017)$ and Cluster 3 imaging phenotype $(H R=0.72,95 \% \mathrm{Cl}: 0.43-0.94, p=0.048)$ were significantly associated with the EFS (Table 3 ). In further analysis, patients, who had Cluster 1 imaging phenotype with controlled blood pressure was set as reference, cases had Cluster 2 imaging phenotype with controlled blood pressure showed better EFS (HR=2.21, 95\%Cl: $1.47-4.32, \mathrm{p}=0.027)$, while those had Cluster 1 imaging phenotype with uncontrolled blood pressure $(\mathrm{HR}=0.64,95 \% \mathrm{Cl}: 0.52-0.89, \mathrm{p}=$ $0.031)$ and Cluster 3 with uncontrolled blood pressure (HR=0.83, 95\% $\mathrm{Cl}: 0.76-0.92, p=0.048)$ suffered worse EFS (Table 3). 
Table 3

Multivariate Cox regression analysis of risk factors associated with events free survival during the followup

\begin{tabular}{|c|c|c|c|}
\hline & HR & $95 \% \mathrm{Cl}$ & P-value \\
\hline \multicolumn{4}{|l|}{ Baseline complications } \\
\hline Renal insufficiency & 0.84 & $0.77-3.18$ & 0.167 \\
\hline Heart dysfunction & 0.89 & $0.81-4.13$ & 0.241 \\
\hline Cerebrovascular events & 0.96 & $0.79-2.14$ & 0.383 \\
\hline Co-exist with severe AR & 0.87 & $0.64-0.95$ & 0.043 \\
\hline \multicolumn{4}{|l|}{ Imaging phenotype } \\
\hline Cluster 1 imaging phenotype & 0.69 & $0.48-0.92$ & 0.017 \\
\hline Cluster 2 imaging phenotype & 1.27 & $0.77-4.21$ & 0.441 \\
\hline Cluster 3 imaging phenotype & 0.72 & $0.43-0.94$ & 0.048 \\
\hline \multicolumn{4}{|l|}{ Baseline hypertensive severity } \\
\hline Mild & 1.79 & $0.78-1.32$ & 0.108 \\
\hline Moderate & 1.03 & $0.82-2.11$ & 0.094 \\
\hline Severe & 0.87 & $0.63-3.18$ & 0.069 \\
\hline \multicolumn{4}{|l|}{ Blood pressure control status } \\
\hline Controlled & 2.13 & $1.32-3.78$ & 0.047 \\
\hline Improved & 1.97 & $0.89-2.31$ & 0.136 \\
\hline Failure & 0.74 & $0.68-1.32$ & 0.087 \\
\hline Cluster $1+$ controlled blood pressure & \multicolumn{3}{|c|}{1 (reference) } \\
\hline Cluster $1+$ uncontrolled blood pressure & 0.64 & $0.52-0.89$ & 0.031 \\
\hline Cluster $2+$ controlled blood pressure & 2.21 & $1.47-4.32$ & 0.027 \\
\hline Cluster $2+$ uncontrolled blood pressure & 1.48 & $0.89-3.11$ & 0.074 \\
\hline Cluster $3+$ controlled blood pressure & 1.13 & $0.91-2.33$ & 0.069 \\
\hline Cluster $3+$ uncontrolled blood pressure & 0.83 & $0.76-0.92$ & 0.048 \\
\hline
\end{tabular}


Kaplan-Meier curves showed that patients with Cluster 1 imaging phenotype might suffer from worse EFS in comparison with Cluster 2 and Cluster 3 imaging phenotype, though this difference was not statistically significant (Fig. 4A). Patients with controlled blood pressure showed better EFS during the follow-up (Fig. 4B).

\section{Discussion}

The present study aimed to summarize the disease characteristics of hypertensive TAK patients and highlight potential determinants to the EFS. We found that: i) about 33\% TAK patients in our cohort suffered from hypertension, among whom, almost half were severe hypertension; ii) three specific imaging phenotypes were identified for hypertensive TAK patients, which could be distinguished from non-hypertensive cases; iii) only $50.8 \%$ patients got controlled blood pressure in the present study and the overall EFS was $67.9 \%$ by the end of a median 48 months follow-up; iv) patients with controlled hypertension showed better EFS, while imaging phenotype also showed effects on the EFS, though not statistically significant.

Previous studies have reported that hypertension occurred in 33-83\% TAK patients, with younger disease onset age (mostly $<40$ years) $[7-10,21]$. One former study even indicated that a combination of hypertension and elevated erythrocyte sedimentation rate (ESR) was useful for diagnosing TAK in patients $<18$ years of age [27]. Our data pointed out that $33 \%$ TAK patients suffered from hypertension, which was consistent with these previous studies. Furthermore, severe hypertension was observed in almost half of the hypertensive cases in our cohort, and severe hypertensive patients were more likely to complain of renal insufficiency and failure to control the elevated blood pressure. These findings call for physicians' awareness of the diagnosis of TAK in young individuals presenting with hypertension, especially in those with indecipherable severe hypertension.

Previous studies have revealed that renal artery stenosis-associated hypertension was observed in about $50 \%$ of TAK cases $[12,27,28]$. In the current study, we also found that the renal artery $(60 \%)$ was the most commonly involved artery in hypertensive TAK patients, and the prevalence of severe and refractory hypertension was significantly higher in patients with renal artery stenosis (data not shown), which might support the important role of renal artery stenosis in the causes of hypertension in TAK. In addition, significant differences of artery involvement was demonstrated between patients with and without hypertension, wherein it was speculated that hypertensive patients might have specific imaging phenotypes. We confirmed this by identifying three specific imaging phenotype clusters in hypertensive patients, which could be distinguished from non-hypertensive cases (Fig. 5). Younger age and worse disease status, especially the prevalence of severe hypertension and renal insufficiency, was observed in patients with Cluster 1 imaging phenotype. What is more, the imaging phenotypes defined in our study also showed significant effects on the EFS. The EFS was significantly lower in Cluster $1(59.6 \%)$ than that in Cluster 2, but similar to that in Cluster 3, which may be related to the higher prevalence of renal insufficiency and persistent refractory and/or malignant hypertension, as well as the lower prevalence of blood pressure control in Cluster 1 and Cluster 3. In addition, although renal and abdominal aorta 
involvement were indicated both in Cluster 1 and Cluster 3, future studies would be needed to determine whether poor prognosis is mainly attributed to this involvement.

Except for renal artery, hypertension in TAK could be caused by multifactorial conditions. In Cluster 2, hypertension might be caused by the involvement of the ascending aorta, thoracic aorta, aortic arch, and its branches instead of the renal and abdominal aorta. Hamida et al. reported that lesions of supraaortic trunks, carotid lesions, and immunosuppressive drugs might contribute to the genesis of hypertension in TAK [29]. Former studies have also found that dysfunctional baroreceptors are possible mechanisms involved in causing hypertension [30]. It is well recognized that a proatherogenic effect occurs in patients with TAK, which may increase arterial stiffness and decrease elasticity of arterial walls that may contribute to elevated blood pressure. In addition, severe AR was observed in 9.3\% hypertensive patients in our study, which was a little lower than that reported in a previous study [21]. Aortic regurgitation may be also associated with hypertension in TAK, and is likely caused by directed valvular lesions, aneurysms arising from the aortic annulus, or annular dilation resulting from extensive dilatational changes of the ascending aorta. Furthermore, we also found that co-existence of severe AR was negatively related to the EFS. Thus, echocardiography monitoring is very necessary for TAK population.

In the current investigation, only $50.8 \%$ cases had blood pressure controlled during the follow-up, which was relatively low. More importantly, patients with blood pressure control showed significantly better EFS. Thus, the main treatment goal for hypertensive TAK patients should be not only to achieve and maintain disease remission, but also to achieve blood pressure control. Combined with the above data, we also made a decision tree diagram using three variables: imaging phenotype, blood pressure control status and co-existence of sever AR (shown as Supplementary Fig S2). Through the diagram, 69.2\% patients could be classified into the right prognosis group. However, the power and accuracy of the decision tree diagram should be validated in the future, due to the small sample size of the present research.

Our study has two major limitations. First, due to the low incidence of TAK, association analyses between severity and controlled status as well as imaging phenotypic categories of hypertension with the prognosis may be underpowered, which warrants future larger studies to validate our results. Second, the follow-up duration was relatively short, and further studies with larger sample size and longer follow-up duration are needed to validate the results.

\section{Conclusions}

In conclusion, 33\% TAK patients suffered from hypertension in out cohort, with almost half severe cases. Three specific imaging phenotypes were identified for hypertensive TAK patients. The blood pressure control rate was $50.8 \%$, with overall EFS of $67.9 \%$ by the end of the follow-up. Our data support blood pressure control status and specific imaging phenotypes showed significant effects on EFS for hypertensive TAK patients.

\section{Abbreviations}


TAK, Takayasu arteritis; AR, aortic regurgitation; EFS, events free survival; ECTA, East China Takayasu arteritis; ACR, American College of Rheumatology; $A B I$, ankle brachial index; SBP, systolic blood pressure; DBP, diastolic blood pressure; MAR, magnetic resonance angiography; CTA, computed tomography angiography; GFR, glomerular filtration rate; SD, standard deviations; IQR, interquartile range; HR, hazard ratio; $\mathrm{Cl}$, confidence interval; $\mathrm{ESR}$, erythrocyte sedimentation rate.

\section{Declarations}

Ethics approval and consent to participate: The study was approved by the ethics committee of Zhongshan Hospital at Fudan University (B2013-115(3)).

Consent for publication: Informed written consent was obtained from each patient.

Availability of data and material: The datasets used and/or analyzed during the current study are available from the corresponding author on reasonable request.

Competing interests: The authors declare that they have no competing interests.

Funding: This study was supported by National Natural Science Foundation of China (NSFC 81771730 and 81801598) and Clinical Cultivation Project of Shanghai Shenkang Science and Technology Development Center (SHDC12019X05).

Authors' contribution: Sun Ying: data analysis and paper written; Wu Sifan and Wang Yujiao: management of electronic database; Chen Rongyi, Huang Qingrong, Ma Lili, and Chen Huiyong: patients follow-up, clinical and imaging data collection; Jiang Lindi: study design.

Acknowledgements: Not applicable.

\section{References}

1. Johnston SL, Lock RJ, Gompels MM. Takayasu arteritis: a review. J Clin Pathol 2002; 55:481-6.

2. Watanabe $Y$, Miyata T, Tanemoto K. Current clinical features of new patients with Takayasu arteritis observed from a cross-country research in Japan: age and sex specificity. Circulation 2015; 132:1701-9.

3. Yilmaz N, Can M, Oner FA, Kalfa M, Emmungil H, Karadag O, et al. Impaired quality of life, disability and mental health in Takayasu's arteritis. (Oxford) 2013; 52:1898-904.

4. Laurent A, Julien H, Nicolas L, Toledano D, Gambotti L, Chalumeau NC, et al. Takayasu arteritis in France: a single-center retrospective study of 82 cases comparing white, North African, and black patients. Medicine 2010; 89:1-17

5. Mwipatayi BP, Jeffery PC, Beningfield SJ, Matley PJ, Naidoo NG, Kalla AA, et al. Takayasu arteritis: clinical features and management: report of 272 cases. ANZ J Surg 2005; 75:110-7. 
6. Park MC, Lee SW, Park YB, Chung NS, Kee SK. Clinical characteristics and outcomes of Takayasu's arteritis: analysis of 108 patients using standardized criteria for diagnosis, activity assessment, and angiographic classification. Scand J Rheumatol 2005; 34: 284-292.

7. Jain S, Kumari S, Ganguly NK, Sharma BK. Current status of Takayasu arteritis in India. Int J Cardiol 1999; 54: S111-S116

8. Park YB, Hong SK, Choi KJ, Sohn DW, Oh BH, Lee MM, et al. Takayasu arteritis in Korea: clinical and angiographic features. Heart Vessels 1992; Suppl 7: 55-59.

9. Zheng D, Fan D, Liu L. Takayasu arteritis in China: a report of 530 cases. Heart Vessels 1992; Suppl $7: 32-36$

10. Mammeri A, Guermaz R, Hatri A, Kessal F, Taharboucht S, Hamrour F, et al. Hypertension during Takayasu's disease: an Algerian single center experience of 279 patients. Annales de cardiologie et d'angeiologie 2017; 66:154-158.

11. Maksimowicz-Mckinnon K, Clark TM, Hoffman GS. Limitations of therapy and a guarded prognosis in an American cohort of Takayasu arteritis patients. Arthritis Rheum 2007; 56:1000- 1009.

12. Sato El, Lima D, Santo BE, Hata F. Takayasu arteritis. Treatment and prognosis in a university center in Brazil. Int J Cardio/ 2000; 75: S163-S166

13. Hong S, Ghang B, Kim YG, Lee CK, Yoo B. Long term outcomes of renal artery involvement in Takayasu arteritis. J Rheumatol 2017; 44: 466-72

14. Sun Y, Dai X, Lv P, Dong Z, Ma L, Yan Y, et al. Characteristics and medium-term outcomes of Takayasu arteritis-related renal artery stenosis: analysis of a large Chinese cohort. J Rheumatol 2021; 48:87-93

15. Petrovic-Rackov L, Pejnovic N, Jevtic M, Damjanov N. Longitudinal study of 16 patients with Takayasu's arteritis: clinical features and therapeutic management. Clin Rheumatol 2009; 28:179185.

16. Kaoru M, Hitoshi O, Junjiro K,Ishibashi-Ueda H, Matsuda H, Minatoya K, Sasaki H, et al. Surgical treatment of aortic regurgitation due to Takayasu arteritis: long-term morbidity and mortality. Circulation 2005; 112:3707-3712

17. VanoliM, Daina E, Salvarani C, Rossi C, Bacchiani G, Schieppati A, et al. Takayasu's arteritis: a study of 104 Italian patients. Arthritis Rheum 2005; 53: 100-107.

18. Arend WP, Michel BA, Bloch DA, Hunder GG, Calabrese LH, Edworthy SM, et al. The American College of Rheumatology 1990 criteria for the classification of Takayasu arteritis. Arthritis Rheum 1990; 33:1129-34.

19. Kerr FS, Hallahan CW, Gordano J, Leavitt RY, Fauci AS, Rottem M, et al. Takayasu arteritis. Ann Intern Med 1994; 120: 919-29.

20. Cifkova R, Erdine S, Fagard R, Farsang C, Heagerty AM, Kiowski W, et al. Practice guidelines for primary care physicians: 2003 ESH/ESC hypertension guidelines. J Hypertens 2003; 21: 1779-86 
21. Qi Y, Yang L, Zhan H, Liang E, Song L, Cai J, et al. The presentation and management of hypertension in a large cohort of Takayasu arteritis. Clin Rheumato/2018; 37: 2781-2788

22. Hata A, Noda M, Moriwaki R, Numano F. Angiographic findings of Takayasu arteritis: new classification. Int J Cardiol 1996; 54: S155-63

23. Bonow RO, Carabello B, de Leon AC, Edmunds LH, Fedderly BJ, Freed MD, et al. ACC/AHA guidelines for the management of patients with valvular heart disease. Executive summary. A report of the American College of Cardiology/American Heart Association Task Force on practice guidelines (Committee on management of patients with valvular heart disease). J Heart Valve Dis 1998; 7(6):672-707

24. Bekeredjian R, Grayburn PA. Valvular heart disease: aortic regurgitation. Circulation 2005; 112:125134.

25. Zoghbi WA, Enriquez-Sarano M, Foster E, Grayburn PA, Kraft CD, Levine RA, et al. Recommendations for evaluation of the severity of native valvular regurgitation with two-dimensional and Doppler echocardiography. J Am Soc Echocardiogr 2003; 16:777-802.

26. Grayson PC, Maksimowicz-McKinnon K, Clark TM, Tomasson G, Cuthbertson D, Carette S, et al. Distribution of arterial lesions in Takayasu's arteritis and giant cell arteritis. Ann Rheum Dis 2012; 71:1329-1334

27. Evan F, Daniel A, Terri F. Hypertension and elevated ESR as diagnostic features of Takayasu arteritis in children. J Clin Rheumatol 2003; 9:156-163

28. Peng M, Jiang XJ, Dong H, Zou YB, Zhang HM, Song L, et al. Etiology of renal artery stenosis in 2047 patients: a single-center retrospective analysis during a 15-year period in China. J Hum Hypertens 2016; 30:124-128.

29. Hmida B, Maazoun, Marzouk, et al. Hypertension in Takayasu disease: Pp.15.72. J Hypertens 2010; 28: e270

30. Milner LS, JacobsDW, Thomson PD, Kala UK, Franklin J, Beale P, et al. Management of severe hypertension in childhood Takayasu's arteritis. Pediatr Nephrol 1991; 5:38-41.

\section{Figures}




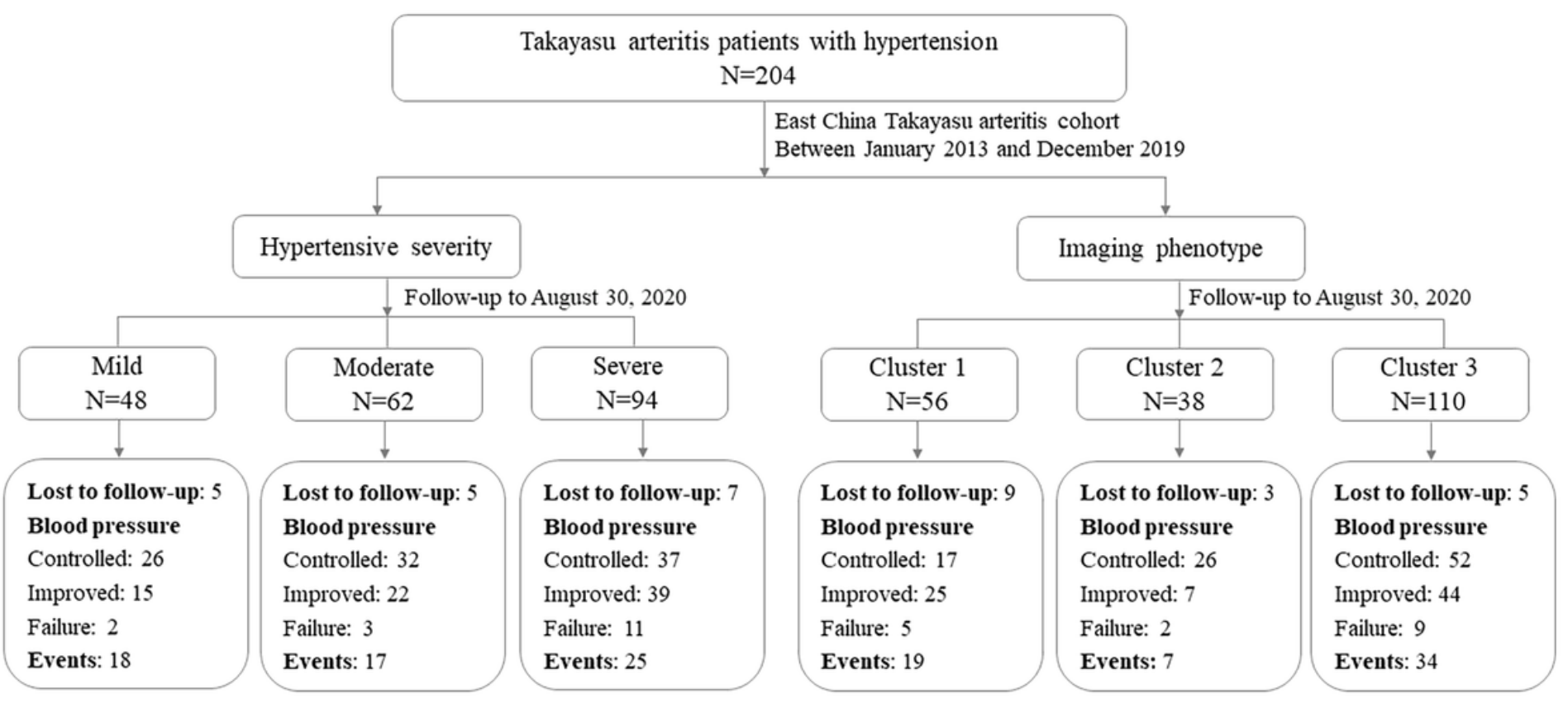

\section{Figure 1}

Study flow chart. In all, 204 hypertensive Takayasu arteritis patients were enrolled in to the present study from the East China Takayasu arteritis cohort between January 2013 and December 2019. Clinical characteristics and imaging features of each case were evaluated. The main outcome of the investigation was the events free survival by the end of August 30, 2020. Subgroup analysis, according to hypertensive severity and imaging phenotype, was also performed. Cluster 1 : involvement of abdominal aorta and/or renal artery; Cluster 2: involvement of ascending aorta, thoracic aorta, and/or the aortic arch and its branches; and Cluster 3: combined involvement of Cluster 1 and Cluster 2.
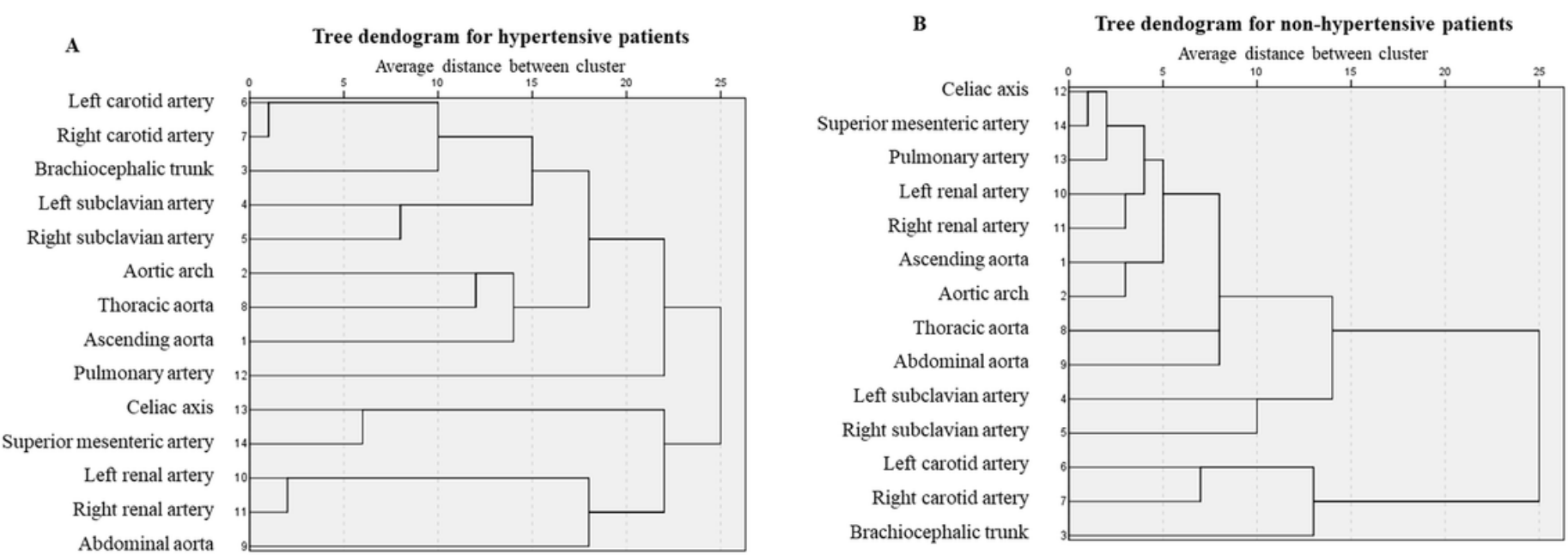

\section{Figure 2}

Tree dendogram for involved arteries of hypertensive and non-hypertensive Takayasu arteritis. Fourteen arteries including bilateral carotid arteries, brachiocephalic trunk, bilateral subclavian arteries, aortic arch, 
ascending aorta, thoracic aorta, pulmonary artery, abdominal aorta, bilateral renal artery, superior mesenteric artery and celiac axis were included in the cluster analysis by a two-step progress to identify imaging phenotypes for hypertensive population. Three specific imaging phenotype clusters was identified for hypertensive patients (A), which could be distinguished from non-hypertensive cases (B).
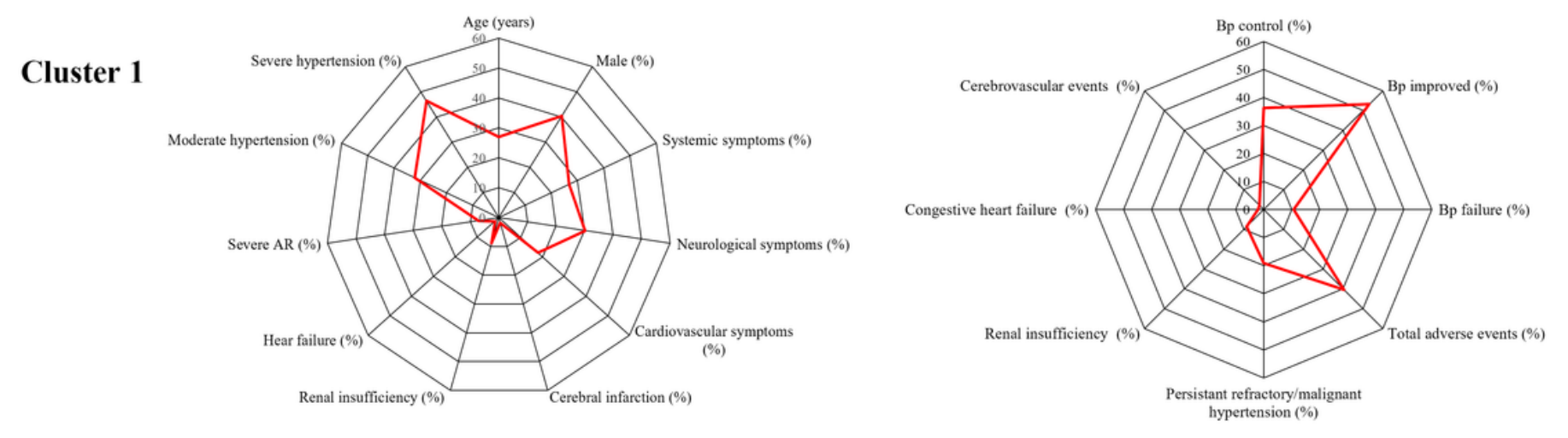

Cluster 2
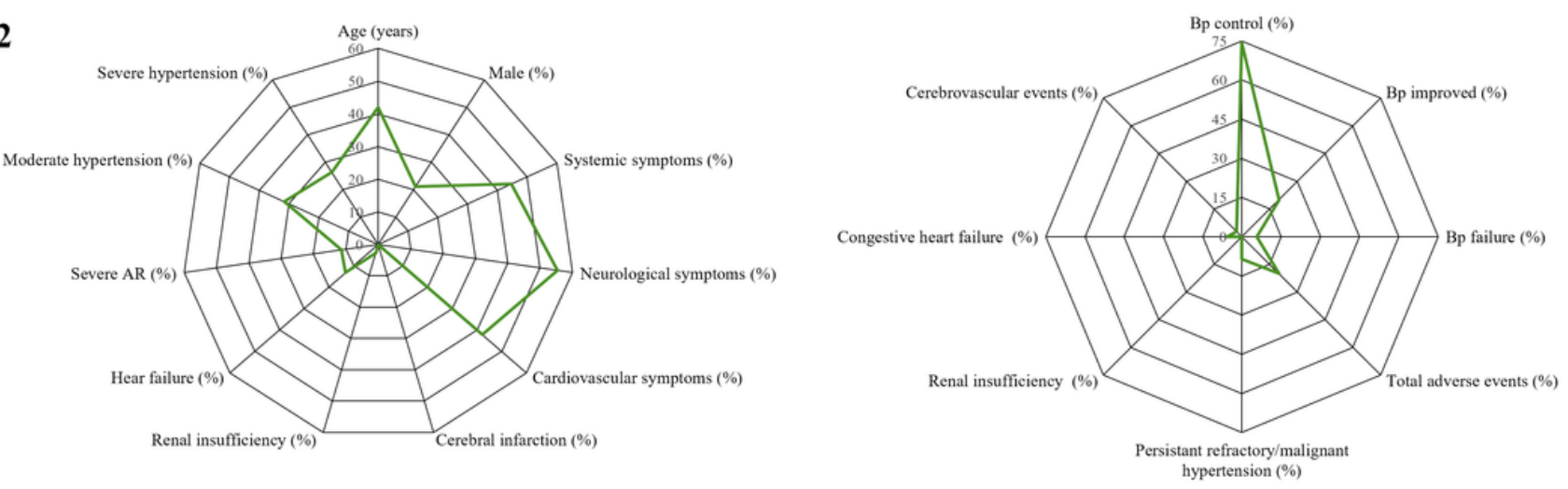

\section{Cluster 3}
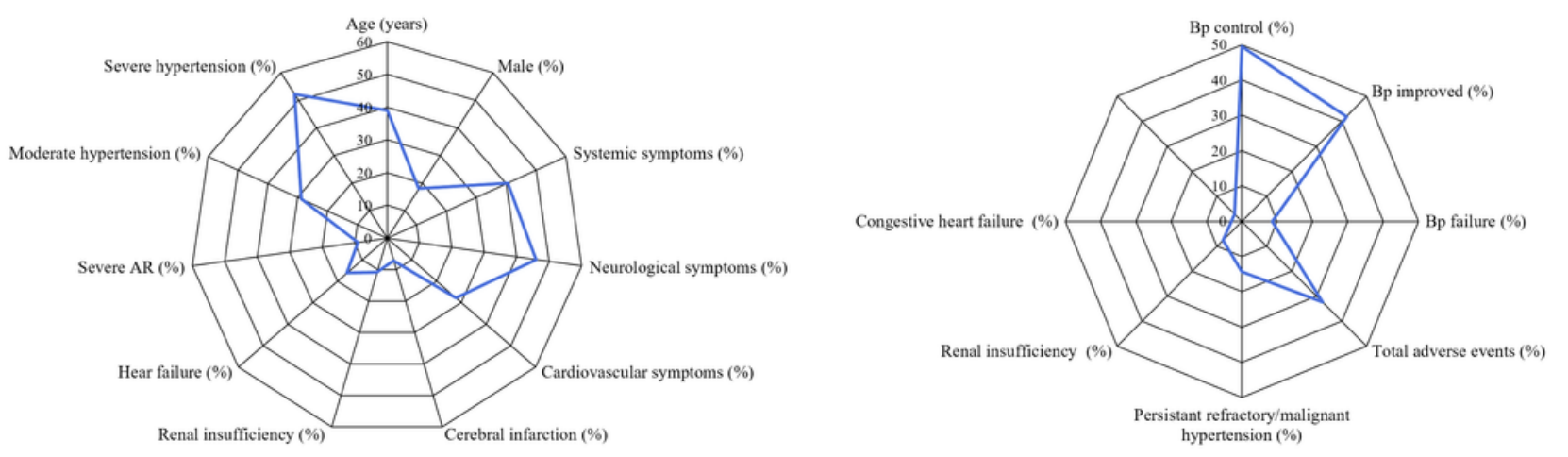

\section{Figure 3}

Clinical characteristics and outcomes of patients with different imaging phenotypes. Clinical characteristics in the radar map (left) included age, sex, clinical manifestations, and baseline complications. Blood pressure control status as well as outcomes, including prevalence of total events, persistent refractory/malignant hypertension, renal insufficiency, congestive heart failure and cerebrovascular events were shown in the right radar map. AR: aortic regurgitation; Bp: blood pressure. 

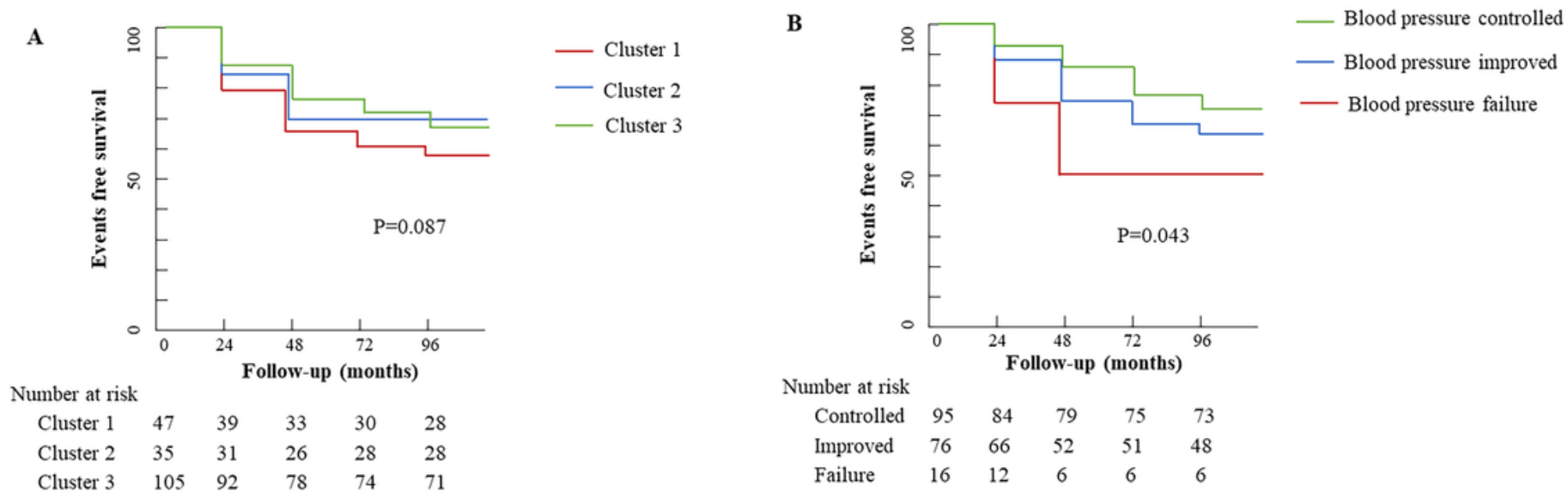

\section{Figure 4}

Events free survival in patients with different imaging phenotypes and with different blood control status. A: Events free survival in patients with different imaging phenotypes. B: Events free survival in patients with different blood pressure control status. Cluster 1: involvement of abdominal aorta and/or renal artery; Cluster 2: involvement of ascending aorta, thoracic aorta, and/or the aortic arch and its branches; and Cluster 3: combined involvement of Cluster 1 and Cluster 2. 


\section{Blood pressure}

\section{Controlled}

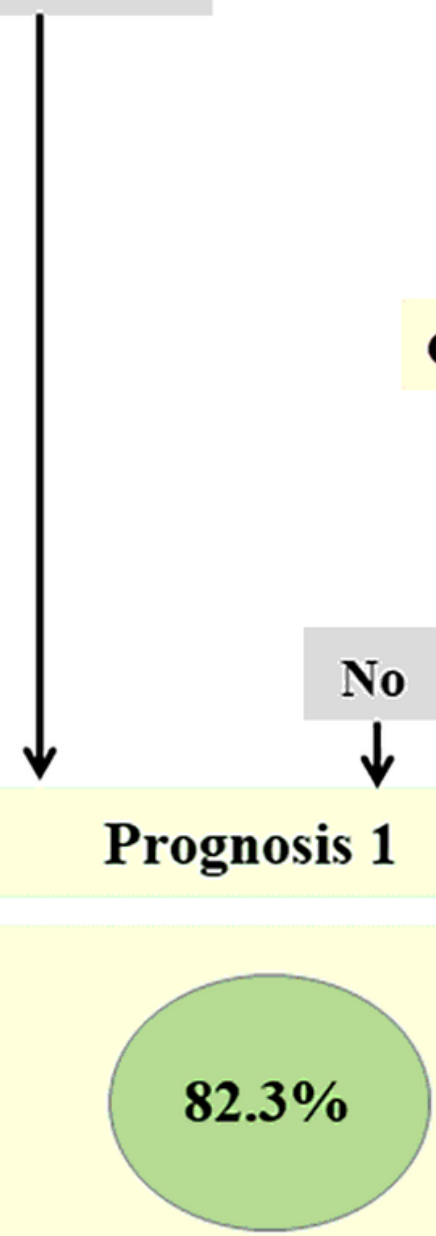

Without events

\section{With events}

\section{Figure 5}

Decision tree for predicting the prognosis of hypertensive Takayasu arteritis. Using three variables including imaging phenotype, blood pressure control status and co-existence of sever AR, a decision tree diagram was established to predict the disease prognosis. Through the diagram, $69.2 \%$ patients could be classified into the right prognosis group.

\section{Supplementary Files}

This is a list of supplementary files associated with this preprint. Click to download. 
- SupplementaryTablesfinal.docx

Page 23/23 